\title{
THE EFFECT OF HIGH FEMORAL OSTEOTOMY ON OSTEOARTHRITIS OF THE HIP
}

\author{
An Anatomical Study of Six Hip Joints \\ Paul D. Byers, London, England \\ From the Institute of Orthopaedics, London and Stanmore
}

Nissen (1966) has recorded the morbid anatomy of the hip joint in a case of osteoarthritis with partial loss of the superior joint space successfully treated by high femoral osteotomy ten months previously. Meachim and Osborne (1970) have described five femoral heads after ineffective operation. There are no doubt differing opinions on how to define a successful osteotomy and by what criteria to recognise it: relief of symptoms, improved movement, reversal of the morbid process (Harris and Kirwan 1964). Moreover, there is probably no unanimity on how long the effect must last before success can be claimed-clearly an important question in studying short-term results.

This paper reports on five hip joints and one femoral head examined from three weeks to eight and a quarter years after osteotomy. In the four patients who survived six months or longer the treatment was regarded as successful.

The diagnosis of osteoarthritis was based on clinical, radiological and laboratory observations. The radiographs indicated loss of articular cartilage from the antero-superior articular surface and associated bone sclerosis. In all but one case there were osteolytic foci in the head and acetabulum. Osteophytes were obvious in all cases, although varying in size.

\section{MATERIAL AND METHODS}

Five of the specimens were whole hip joints obtained by necropsy; one was the upper part of the femur removed at operation. The specimens were fixed in formalin and then studied by gross inspection and microscopical sections. All material was cut in 3-millimetre slabs, radiographed, then decalcified in E.D.T.A. and processed to paraffin blocks. The sections were stained with haematoxylin and eosin, but special stains were used as indicated.

\section{RESULTS}

Macroscopic observation and fine detail radiographs of all hip joints showed features of osteoarthritis. In each instance the head showed evidence of loss of bone from the superior surface and the addition of new bone on the inferior surface. In some the fovea was identified, and these had osteophytes of varying size; in others the fovea was absent. Marginal osteophytes of variable size were present in all.

There are of course wide variations in the appearances of femoral heads resected in the treatment of osteoarthritis by prosthetic replacement. As yet there is no classification of these appearances and their significance is open to interpretation. A personal judgment on these six hip joints in the light of an experience of more than 1,500 resected femoral heads suggests that they are roughly comparable and that they are likely to have been of a type in which the area of exposed bone is not widely occupied by foci of fibrous tissue protruding from the subchondral marrow. This is an important assessment, for it clearly affects the validity or usefulness of putting these cases into a time series following osteotomy, as done here.

The acetabula showed varying degrees of outer marginal and foveal osteophytes, sometimes to the point of producing a deep acetabulum with no identifiable fossa. The labrum was generally ragged or replaced by osteophytes.

VOL. 56 B, NO. 2, MAY 1974 
Exposed bone, or the site where it has been exposed, was usually readily identified in both the heads and the acetabula. The evidence clearly indicated that after osteotomy soft tissue covers this. Details of the mechanism whereby this occurs is the predominant purpose

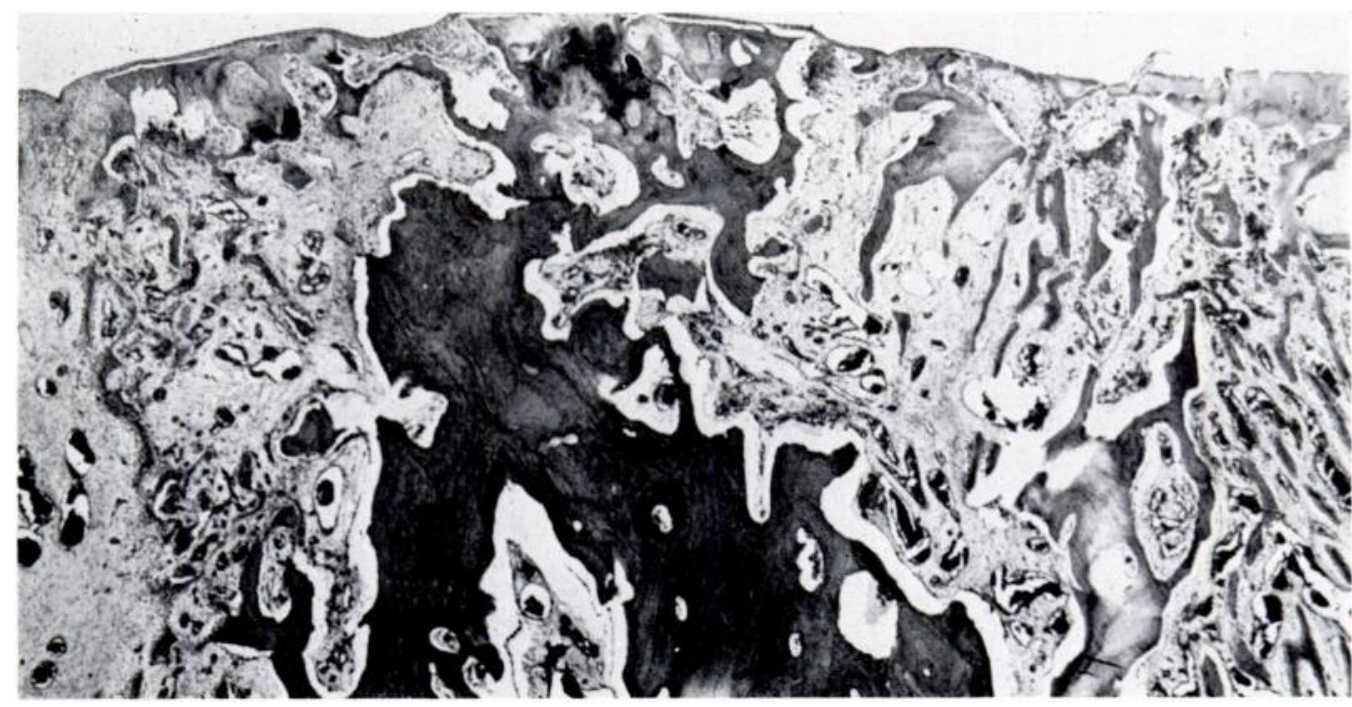

Fig. 1

Case 1 (three weeks) - Showing loss of articular cartilage from the femoral head except on the right, where it is thin and frayed; bone sclerosis in the mid region; and fibrous osteolytic foci. The latter are vascular, and new bone trabeculae extend into them. The exposed bone is covered by a thin membrane. (Haematoxylin and eosin, 20 .)

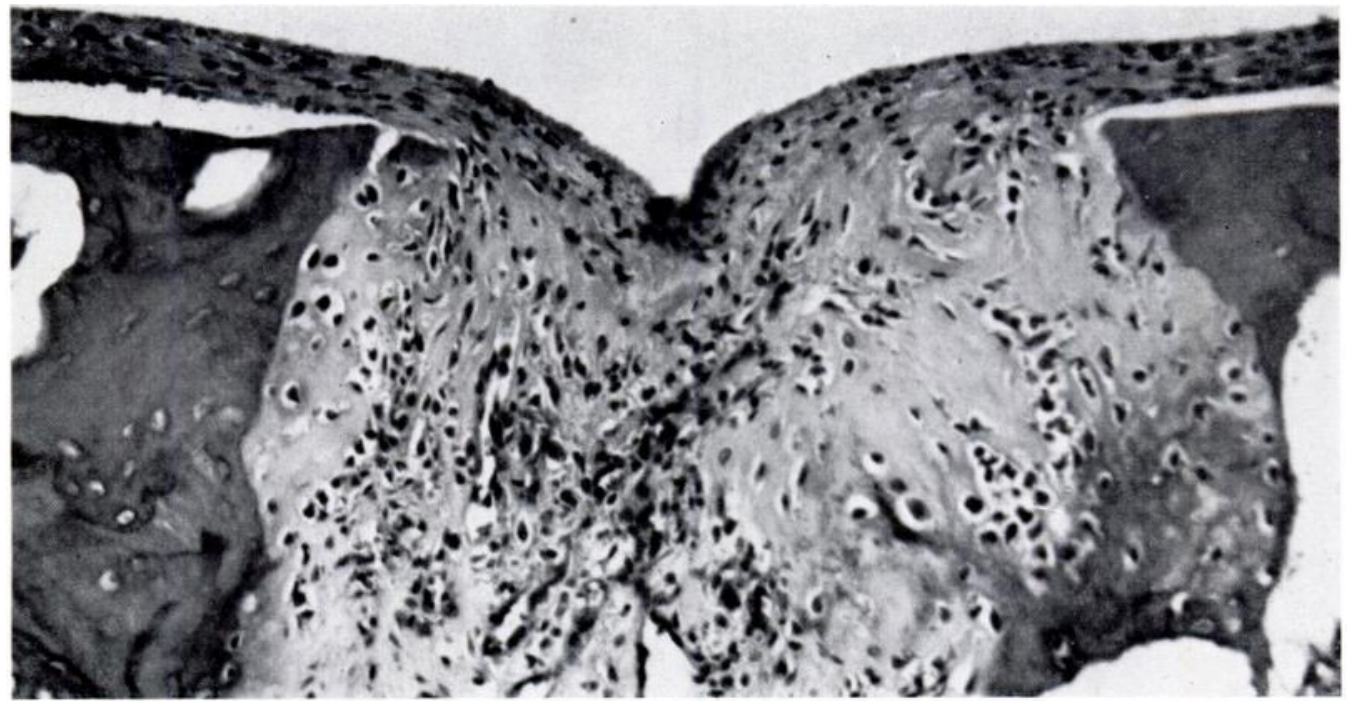

FIG. 2

Case 1-Showing a small fibrous focus at the surface of the exposed bone from which arises a fibrocellular membrane, overlying but not attached to the adjacent bone. The fibrous focus contains blood vessels and shows chondroid and bony differentiation. (Haematoxylin and eosin, $>160$.)

of this paper. But bone sclerosis and osteolytic foci will also be commented upon because these diminished or disappeared after osteotomy.

\section{CASE REPORTS}

Case 1-Three weeks after osteotomy-This woman, aged sixty-six, had pain in the right hip for four years, gradually increasing and becoming constant. She limped but walked any required distance. 
Joint movement was slightly reduced. Radiographs revealed early changes of osteoarthritis in the left hip and a marked spondylolisthesis at L.5-S.1. A latex test was negative and the erythrocyte sedimentation rate was 14 millimetres in the first hour. The patient died of pulmonary embolism twenty-two days after intertrochanteric osteotomy.

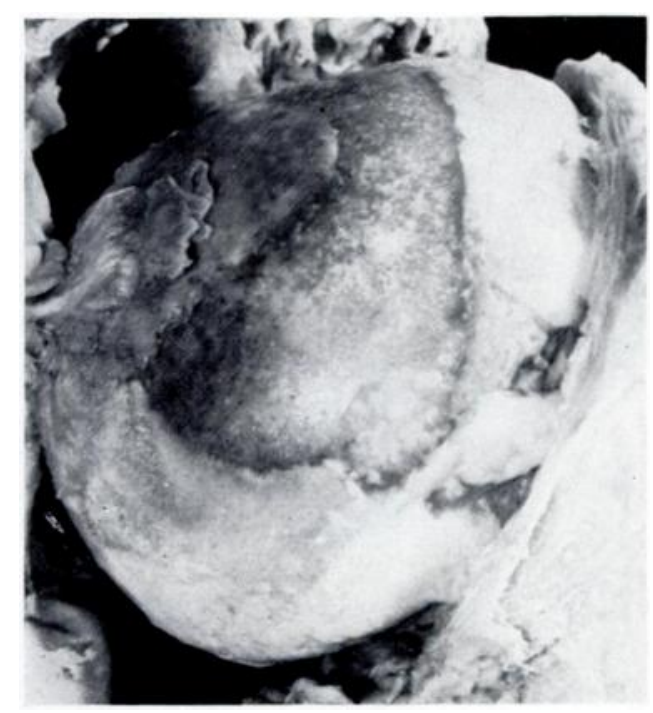

Fig. 3

Case 2 (ten weeks)-A photograph of the femoral head showing an area of smooth sclerotic exposed bone, the medial part of which is covered by a crescent of soft tissue.

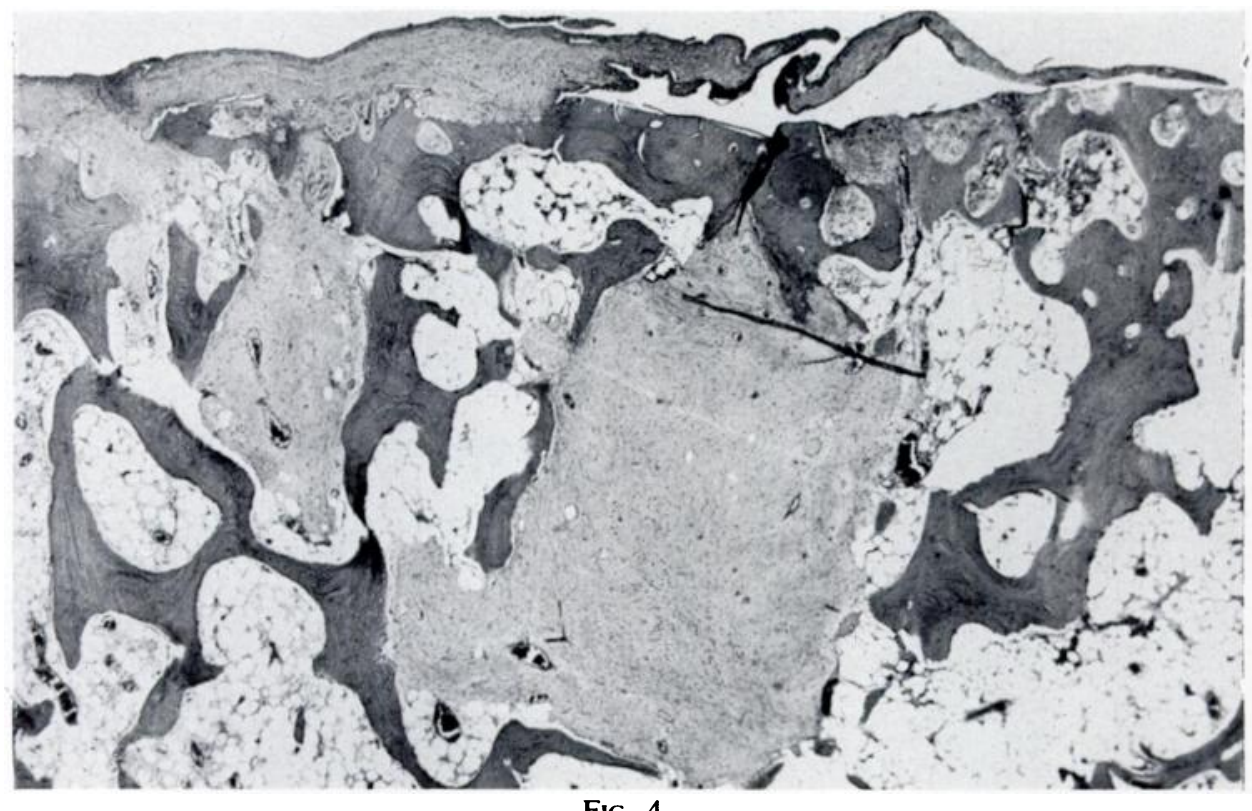

Fig. 4

Case 2-On the left of the section the newly formed fibrous membrane is attached to fibrous pockets in the bone and to some bone trabeculae. The subchondral region is being actively remodelled and contains two small solid fibrous foci. Towards the right the membrane becomes thinner; its leading edge is composed of fibrin. (Haematoxylin and eosin, $\times 20$.)

Morbid anatomical features-In the antero-superior part of the head there was a clearly demarcated area of exposed eburnated bone through which protruded scattered small foci of soft tissue. The surrounding articular cartilage was rough. Inspection of the divided specimen 


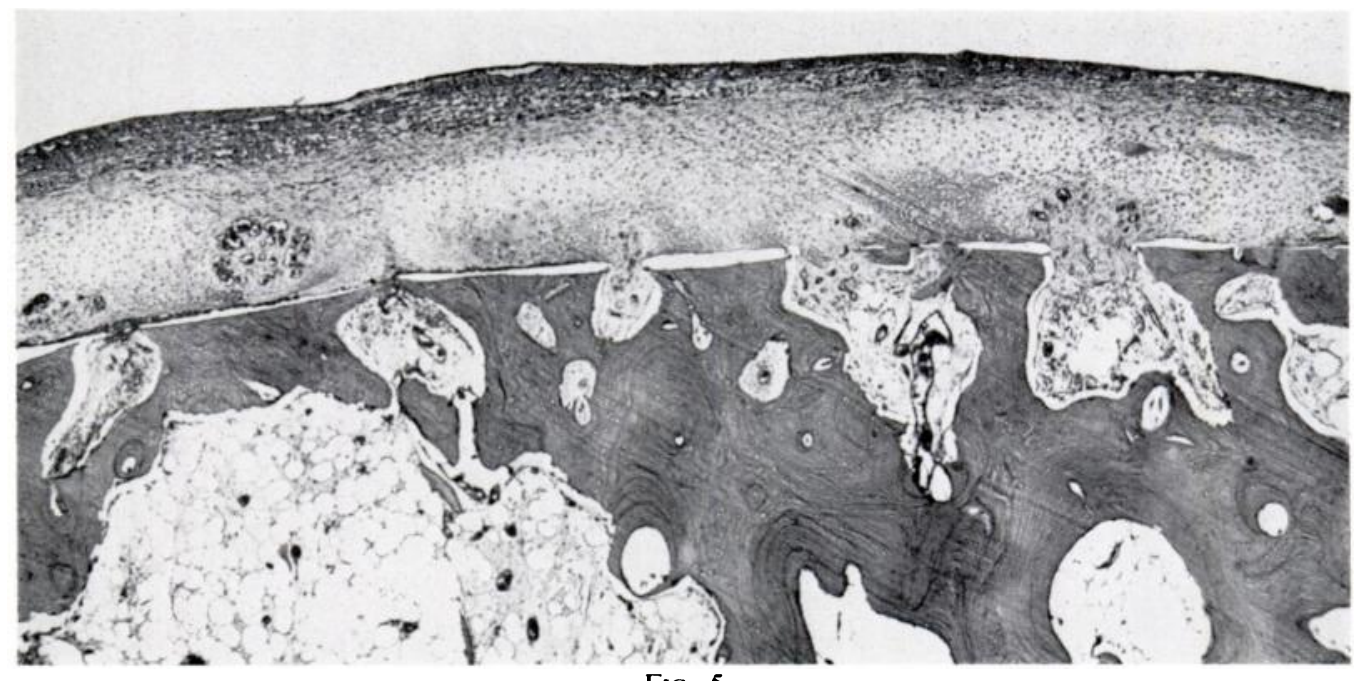

FIG. 5

Case 2 (ten weeks) - The mid region of the fibrous membrane is attached only to marrow fibrous foci and merely overlies the exposed bone. It contains clusters of capillaries and patches of immature bone, and there is chondroid differentiation. The surface is covered by fibrin. (Haematoxylin and eosin, 25.)

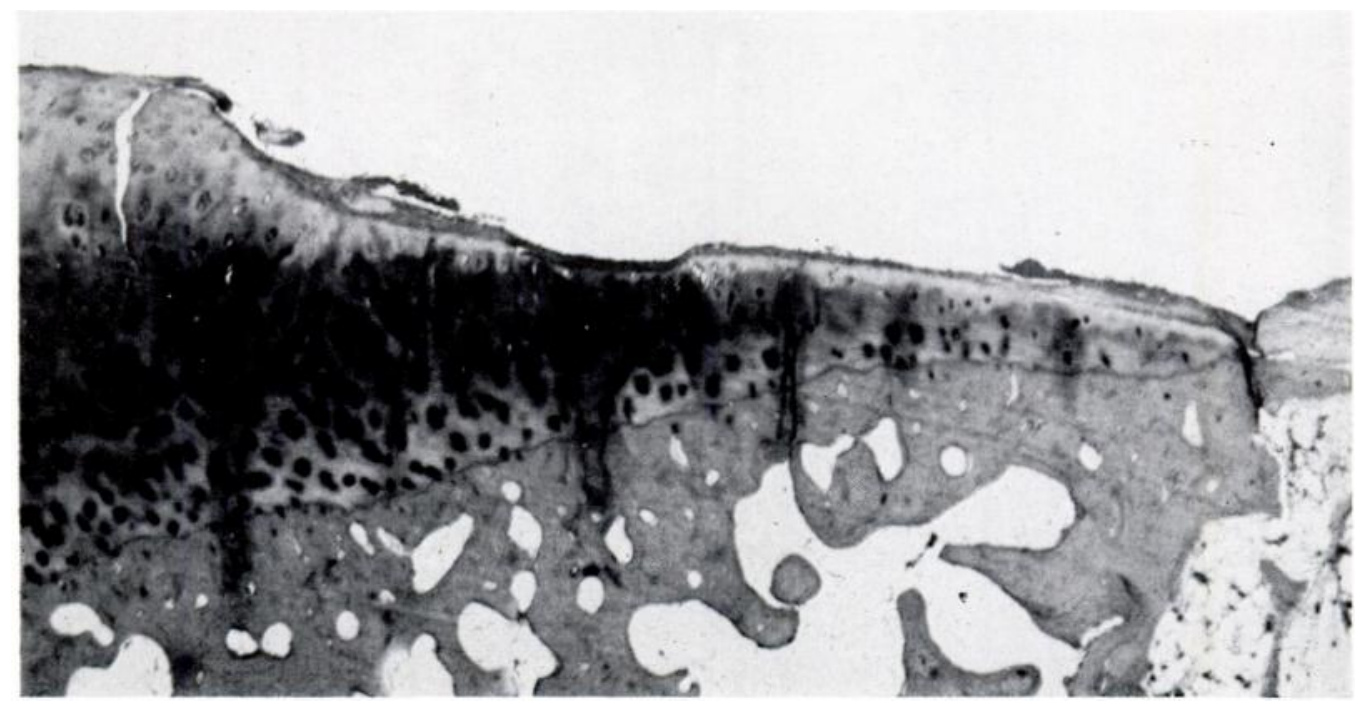

FIG. 6

Case 2-Here the fibrous membrane overlaps the pre-existing cartilage, which thins abruptly to the bone level. The smooth undulating cartilage surface suggests that some of it has been resorbed (Haematoxylin and eosin, 25.)

and fine detail radiographs showed subchondral osteolytic foci measuring up to 10 millimetres in diameter. These were confined to the region of vertical trabeculae arising from the calcar. A comparable area of bone exposure was present in the acetabulum, but with rather more soft tissue protruding from it.

The usual changes of osteoarthritis were present in histological sections from both the head and acetabulum, that is, frayed articular cartilage becoming progressively thinner and disappearing at the point where it met exposed sclerotic bone, in which there were fibrosis of marrow and pockets of soft tissue reaching the surface. There were several large subchondral 
fibrous foci without any cysts in them. Numerous dilated thin-walled blood vessels were a striking feature. Osteoblastic activity was intense, with fingers of newly formed membranous bone extending into the focal fibrous lesions (Fig. 1). The surface of the exposed bone was covered by either a fibrinous acellular material or a very thin fibrocellular membrane (Figs. 1 and 2) attached not to the bone itself but to the minute pockets of fibrous tissue in it. All the appearances suggested that the fibrin appeared first and provided a supporting network for cells migrating from the points of attachment. Even though the membrane extended up to, and in some places over, the adjacent degenerated articular cartilage, it was not invaded by any cells there.

Case 2-Ten weeks after osteotomy-This woman, aged sixty-two, had progressive discomfort of the left hip for four years. She limped but was able to cut her toe nails and walk up to one mile. Movement of the joint was slightly limited. The erythrocyte sedimentation rate was 17 millimetres in the first hour. Radiographs showed moderately advanced osteoarthritis with loss of the superior joint space and sclerosis of the subchondral bone. She died from pulmonary embolism ten weeks after varus osteotomy.

Morbid anatomical features - In the antero-superior region of both the head and the acetabulum there were extensive corresponding areas of exposed bone, of which a part was covered by a membrane of soft tissue but which otherwise was smooth and shiny with a few very small protruding soft-tissue foci (Fig. 3). Histological sections of the fibrous membrane showed it extending from one to another of the superficial soft-tissue foci in the sclerotic bone (Figs. 4 and 5) without attachment to the underlying bone per se. Its deep aspect was invaded by blood vessels, and bands of bone were forming a new subarticular bone plate (Fig. 5). Chondroid differentiation had occurred here and there, generally superficial to the forming bone (Fig. 5). The surface was covered by fibrin. The edge of the fibrous membrane extended freely over exposed bone; the level of the adjacent bone, to which it had become attached, had been reduced by remodelling (Fig. 4). In the other direction the fibrous sheet either blended with or overlapped the residual articular cartilage for a short distance (Fig. 6). Here the surface of the cartilage was not fibrillated as it would be in osteoarthritis, and its surface was lower than expected and irregular-all observations that suggested loss of cartilage caused by the fibrous tissue.

Comparison of clinical radiographs, slab radiographs and histological sections indicated that there had been considerable resolution of osteolytic foci. However, in the sclerotic exposed bone there were still small fibrous, and sometimes cartilaginous, foci which protruded on to the surface. Remodelling of the subchondral bone was evident and had been responsible for the reduction in bone sclerosis (Fig. 4).

Case 3-Ten months after osteotomy - This man, aged fifty-three, had increasing pain and disability from both hips for ten years. A cup arthroplasty had been performed on the left hip eight years previously and was deteriorating. Over the eighteen months before osteotomy the right hip became much worse. He sat and dressed with difficulty and walked 200 to 700 yards with the aid of a stick. He had advanced degenerative changes in the lumbo-sacral region. Radiographs showed typical osteoarthritis of the right hip at a relatively early stage, with incomplete loss of the superior joint space. Nine months after osteotomy he was very pleased with the results; he was able to walk two miles and to put on his shoes easily. Radiographs now showed a widened superior joint space. He died ten months after the osteotomy from primary carcinoma of the liver while he was still in hospital after a Girdlestone revision of the left cup arthroplasty. This case has been reported in some detail by Nissen (1966), who found evidence of regeneration of the weight-bearing articular cartilage.

Morbid anatomical features-No region of exposed bone was found in either the head or the acetabulum. The antero-superior articular tissue was not greatly different in appearance from the remainder but might have been the site of bone exposed by the disease process. Fine detail radiographs of both the head and acetabulum showed virtually no subchondral bone sclerosis, a well formed subchondral bone plate, and a few osteolytic foci that appeared to have undergone some resolution.

vOL. 56 B, NO. 2, MAY 1974 


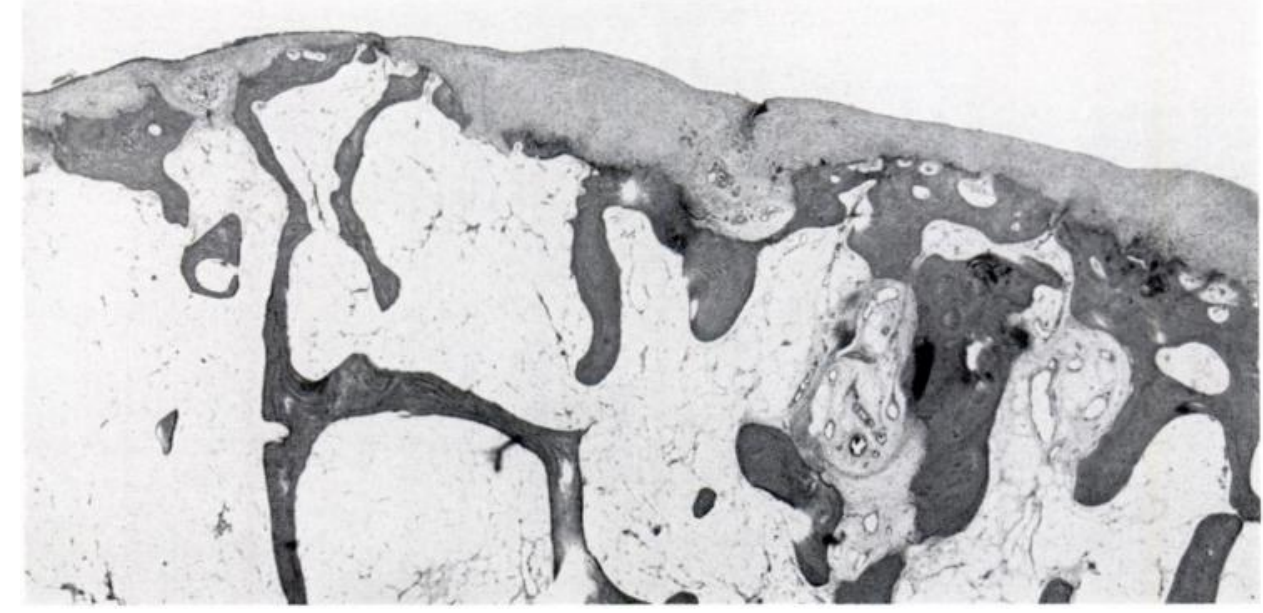

FIG. 7

Case 3 (ten months)-Showing a thin fibrocartilaginous covering on the antero-superior part of the femoral head. There are small foci of marrow fibrosis and a partially formed subchondral plate. (Haematoxylin and eosin, 20.)

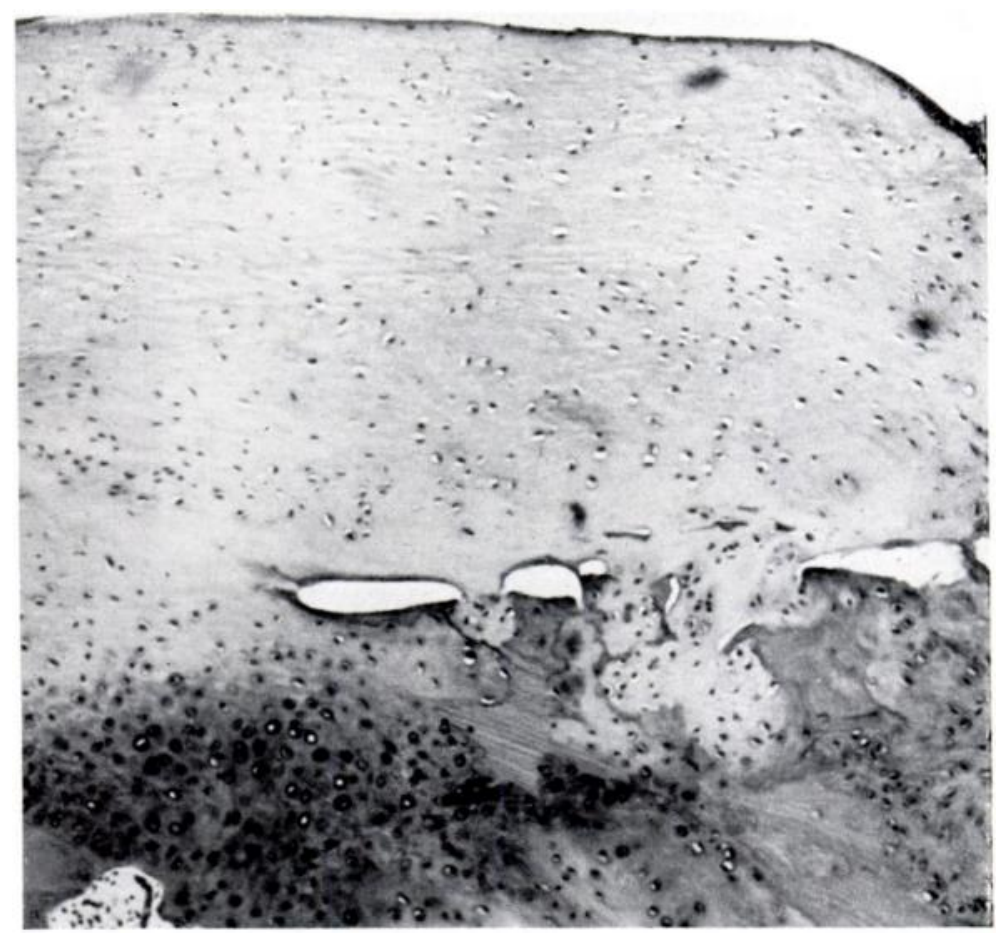

Fig. 8

Case 3-Showing clefts in the fibrocartilaginous membrane where it is incompletely attached (see also Figure 5). (Haematoxylin and eosin, $\times 60$.)

All the histological sections confirmed the absence of exposed bone, the site now being covered by fibrocartilaginous tissue of a variable thickness, with some areas clearly hyaline in nature and others more fibrous (Figs. 7 to 10). The underlying bone and marrow had been extensively remodelled, although there was still some marrow fibrosis (Fig. 7). There were several clefts between the membrane and the bone where attachment had not been completed (Fig. 8). 


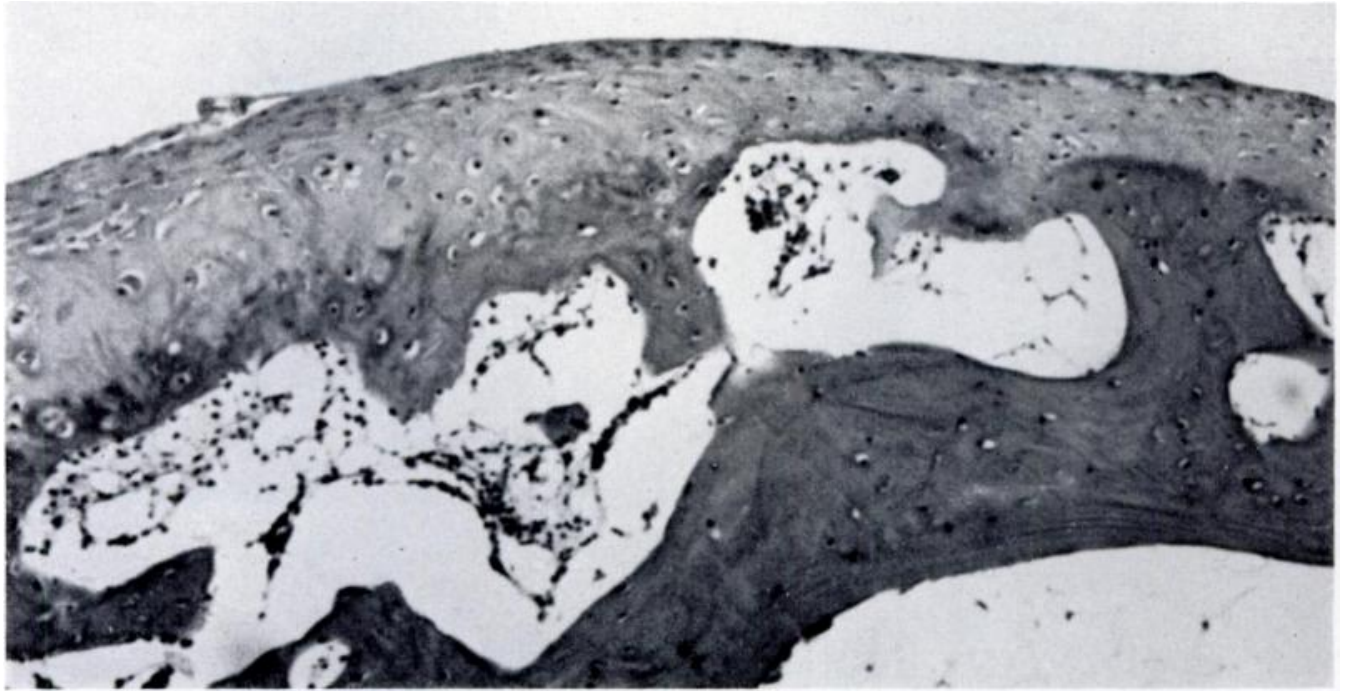

Fig. 9

Case 3 (ten months)-A higher power view of the fibrocartilaginous membrane with a thin subchondral plate of immature bone. (Haematoxylin and eosin, 60.)

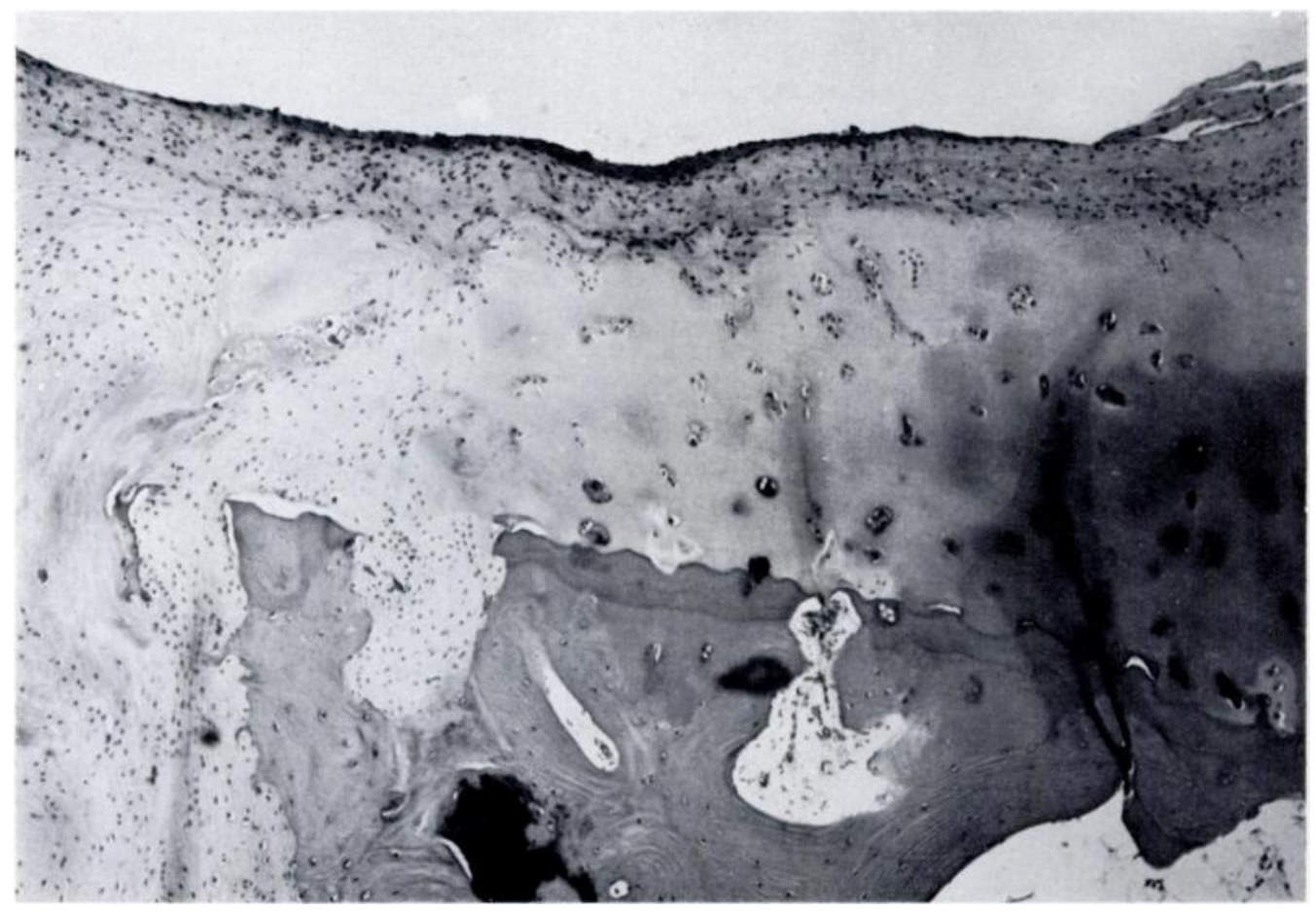

Fig. 10

Case 3-Showing pre-existing articular cartilage covered by a fibrous membrane and in a poor state of preservation. (Haematoxylin and eosin, $\times 60$.)

Although the macroscopic identification of the re-covered area was difficult the margin could be judged histologically. Figure 10 shows the boundary; the fibrous mantle covers the pre-existing cartilage, which is reduced in thickness.

Case 4-Right hip joint four and a half years after osteotomy - This woman, aged forty-eight years, had pain in the left hip for eighteen months, a limp and greatly reduced joint movement. Radiographs showed 
loss of the superior joint space, bone sclerosis and slight lateral subluxation. Osteotomy gave lasting relief of pain except after prolonged standing. The joint space widened after three months and this was maintained, but osteophytes slowly increased. The morbid anatomical features of this left hip after eight years are described later.

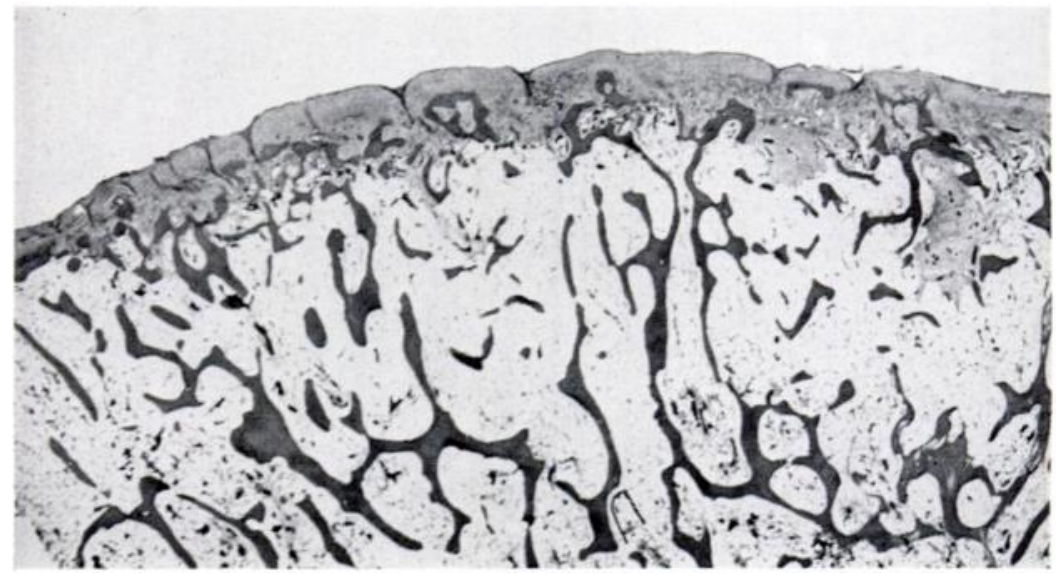

Fig. 11

Case 4 (four and a half years)-There is a relatively normal subchondral bone architecture but no complete bone plate. (Haematoxylin and eosin, 7.)

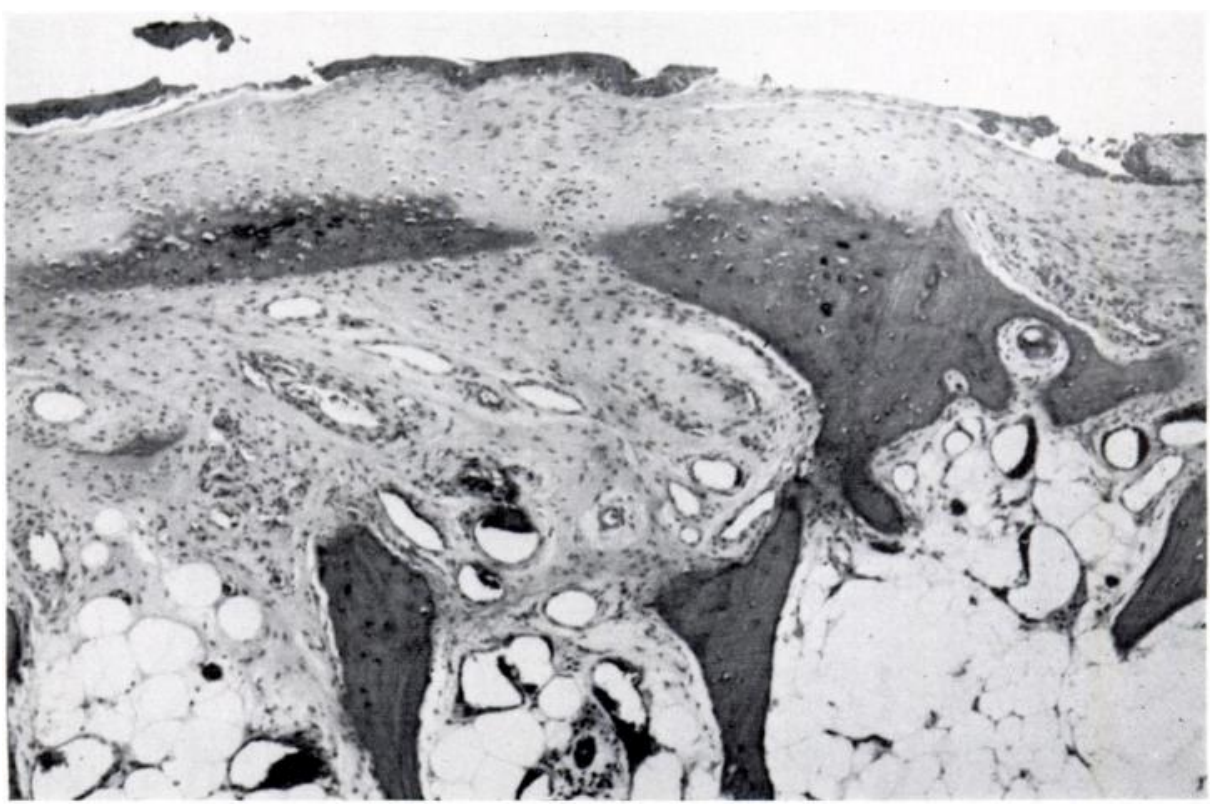

Fig. 12

Case 4-An enlarged view of Figure 11. A fibrocartilaginous membrane overlies the bone and marrow, which is fibrotic and vascular. There is bone formation in the depths of the membrane: its surface is covered by fibrin. (Haematoxylin and eosin, 40 .)

The right hip joint space was slightly reduced when she was first seen and there was a slowly progressive reduction of it accompanied by osteophyte formation and sclerosis of the acetabular roof. Three years and ten months after the left osteotomy, the same operation was performed on the right side. This relieved the pain but did not reduce a slight subluxation or lead to obvious radiographic increase in the joint space. The osteophytes continued to increase.

From the outset the disease was confined to the hips. Neither the erythrocyte sedimentation rate nor the rheumatoid factor was tested. 
Morbid anatomical features-The site of former bone exposure had been completely re-covered by dark granular tissue. Fine detail radiographs did not show a recognisable subchondral plate in this area, but demonstrated minimal sclerosis in the head, more widespread but not very dense sclerosis of the acetabulum, and resolving osteolytic foci in both.

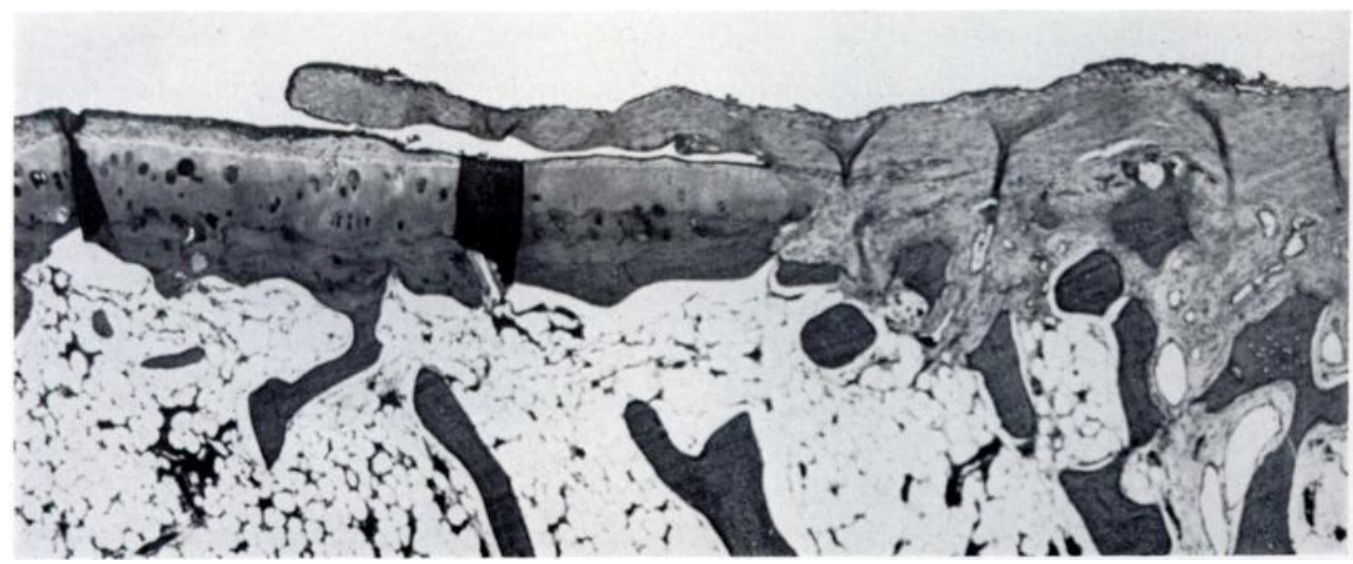

Fig. 13

Case 4 (four and a half years)-The appearances at the juncture of the fibrous membrane and pre-existing cartilage indicate that cartilage and subchondral plate have been resorbed. A free tongue of membrane extends towards the left over the cartilage. Beyond this there is a fibrocellular layer on the surface of the articular cartilage. (Haematoxylin and eosin, $\times 30$.)

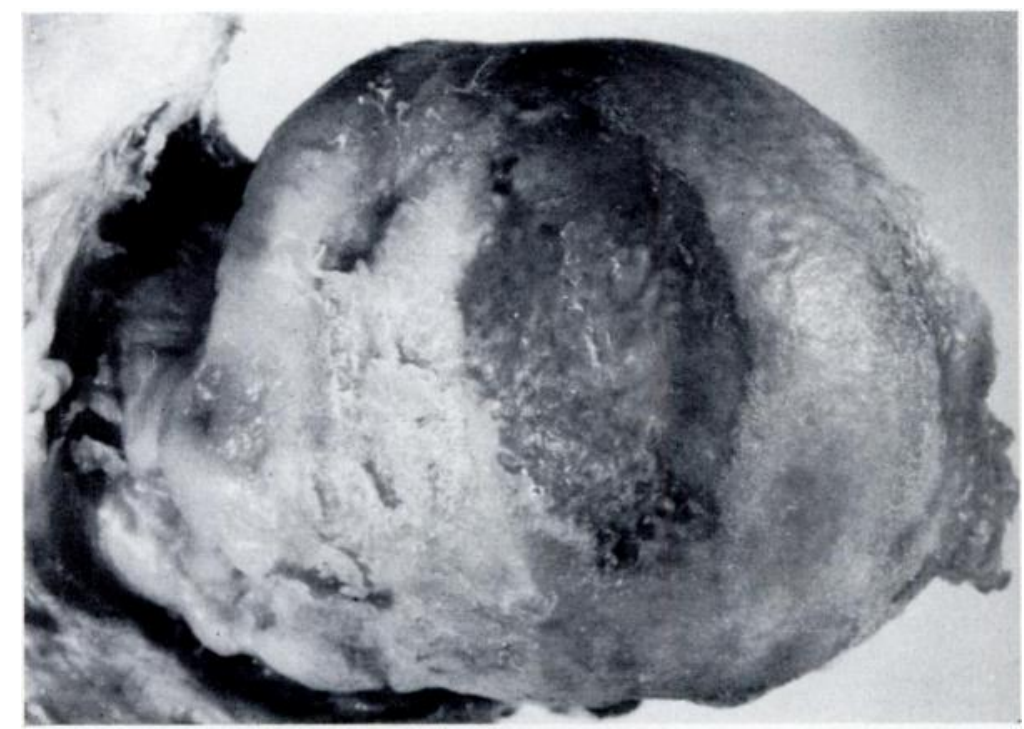

Fig. 14

Case 4 (eight years)-The site of exposed bone has been covered by dark, soft tissue. A comparable re-covered area was present in the acetabulum. Within both areas a small area of bone was still exposed.

Histological sections showed that the surface originally denuded of articular cartilage was now covered by fibrous tissue (Fig. 11) which was moderately cellular, highly collagenous and sometimes vascularised on its deeper surface, where new formed bone occurred (Fig. 12). Pre-existing bone underlying this vascularised fibrous tissue was being resorbed and, at least in some places, the articulating surface was at a slightly lower level. This difference in level 
was measurable in microns, so that the position was relatively stable. Where the membrane formed in response to the osteotomy abutted against the pre-existing cartilage and its subchondral plate, both were resorbed (Fig. 13, cf. Fig. 10 also). There was no knowing how much tissue had been or would have been lost this way. Adjacent articular cartilage might be overlapped by the fibrous membrane, which was not always attached to it (Fig. 13); on the other hand fibrous tissue at the surface of the cartilage did not always appear to derive from the fibrous membrane and could have been the result of metaplasia of surface cells (Fig. 13). When the adjacent cartilage was not covered by the fibrous membrane it tended to be thin, poorly stained and fibrillated.

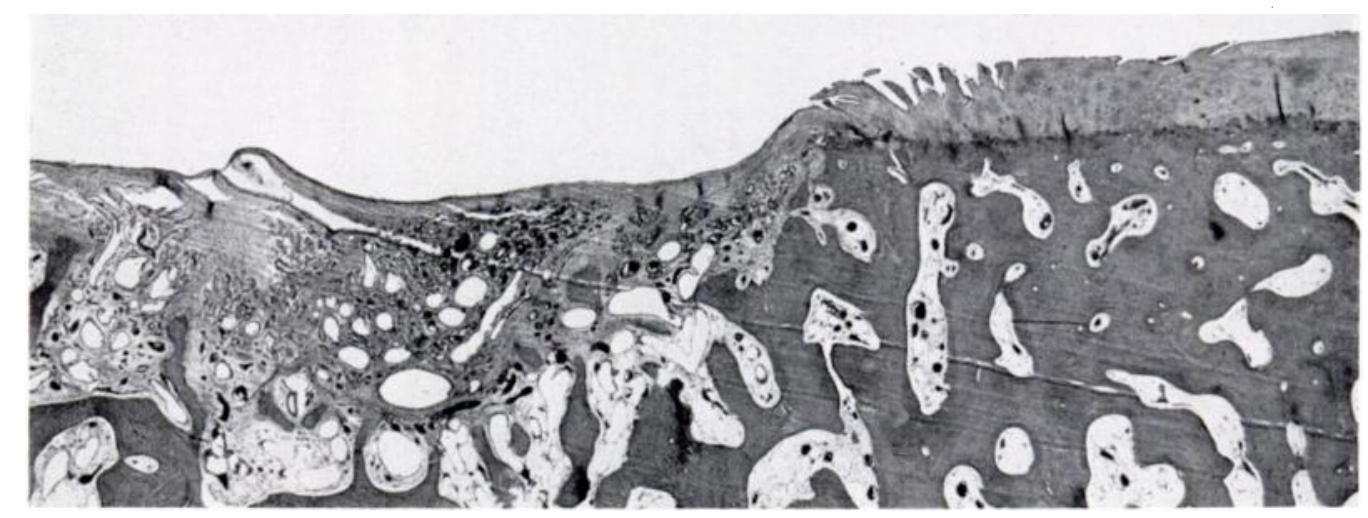

Fig. 15

Case 5 (eight and a half years)-Showing the fibrous membrane at a slightly lower level than the fibrillated articular cartilage. (Haematoxylin and eosin, 20.)

Case 5-Six and three-quarter years after osteotomy-This man, aged fifty-nine, had pain and stiffness of the left hip for three years. Radiographs showed moderately advanced osteoarthritis of the left hip, with loss of joint space at the apex and medially, and sclerosis of the head and acetabulum superiorly, but no osteolytic lesions. Osteophytes were only moderately large. Osteotomy was carried out. Despite non-union, which caused him pain, the joint improved. Six years after the osteotomy he could barely walk because of deformity of the left leg due to persistent non-union and also because he had developed osteoarthritis of the right hip. Radiographs at this time showed advanced degenerative disease in the lumbo-sacral region and in the right hip joint, with gross loss of joint space, "cyst" formation and sclerosis, but normal hands and feet. The left hip now had quite large acetabular osteophytes, little evidence of sclerosis but no "cysts" and a uniformly wide joint space. Six and three-quarter years after the osteotomy total replacement of the left hip joint was performed and a specimen of the upper end of the femur obtained.

Morbid anatomical features-Only the upper end of the femur was available following the total hip replacement. Large osteophytes were present on the outer margin of the head. The whole articulating surface was covered by soft tissue, clearly cartilaginous inferiorly but less easy to define on the superior surface where it was of irregular texture and colour. The area of re-formed tissue could not be identified with certainty. Fine detail radiographs of a few slices showed normal bony tissues without evidence of osteolytic foci. Such foci were not evident in clinical radiographs before osteotomy, whereas bone sclerosis had been. Histologically all but the thinnest covering tissue was either hyaline or fibrocartilage but nowhere was the former perfectly normal, showing variable reduction in staining and in the number and order of cells, and fibrillation. Although the thinnest material was fibrous it nevertheless overlay a normal subchondral bone plate. None of the sections showed appearances akin to those of other cases where regenerated tissue covered bone previously exposed. Possibly the sections had not been taken from the right areas. Unfortunately the specimen had been discarded and further study was precluded. 
Case 4-Left hip eight and one-quarter years after osteotomy-The history of this case has already been given.

Morbid anatomical features-Large interlocking osteophytes prevented all movement of the hip and had entirely obliterated the fovea and fossa. On the antero-superior surface of the head (Fig. 14) and the acetabulum was a large dark area of soft tissue which enclosed a small area of exposed bone on the head and a minute one in the acetabulum. The surrounding articular cartilage was smooth but finely pebbled. Fine detail radiographs showed sclerosis where bone was exposed, but no osteolytic foci.

Histological sections of the re-covered exposed bone were remarkably like comparable sections of the right hip. Fibrous tissue overlay the bone, had a layer, often very thick, of fibrin on its surface and a marked vascular component in its depth, and contained foci of bone formation in its substance. Underlying bone had been remodelled with a slight consequent subsidence of the surface (Fig. 15). Adjacent cartilage which had been covered by fibrous tissue seemed to be resorbed and replaced by it. It was not possible to determine if the exposed bone within the fibrous tissue was already exposed at the time of osteotomy or had been re-exposed since then.

\section{CONCLUSIONS AND DISCUSSION}

A study of six hip joints can hardly be expected to elucidate all the events that take place after osteotomy or even to bring forward all the questions that need to be asked. A basic question is the state of the joint at the time of osteotomy. Nissen (1971) suggested that in less advanced cases still with some joint space there is a residual layer of healthy cartilage cells which forms a base for reparative activity. It may be that osteotomy is sometimes performed when no bone has been completely exposed, but the evidence for this is indirect whereas the direct evidence of the two early cases of this study indicate the opposite. The following discussion starts from the premise that bone is exposed at the time of osteotomy.

The effect of osteotomy appears unquestionably to be the formation of a mantle of soft tissue that protects the bone against further destruction. Fibrin provides the scaffolding by which cells migrate over the exposed bone. In every area of bone exposure there are at least microscopic pockets of soft tissue at the surface, which may be fibroblastic and/or chondroid in character. Whether these cells derive from the cartilage or the marrow is arguable, but they are undoubtedly the source of the cellular element in the repair process. Evidence for other origins for the cells-the synovium and surrounding articular cartilage-has been found wanting. The initial attachment of the new mantle is to the fibrous foci in the sclerotic bone. Its attachment to the whole surface comes about by remodelling, during which cells lay down collagen fibres between the two. These observations are similar to those made by Bentley (1974) in animal experiments.

Simple cellular division is adequate to account for further development of the mantle. Nevertheless, fibrin was present on the surface in several cases years after the osteotomy: it is not necessary to attribute to this the continuing role of a scaffold for advancing cells. Maturation of the mantle is clearly a variable, complex and uncertain process dependent on cellular differentiation and intercellular matrix production. Bone formation occurs in its deeper layers not by enchondral ossification but by membrane bone formation; nevertheless it is at sites where capillaries have penetrated. This new bone formation can lead, it seems, to a fairly complete restoration of the subchondral plate.

Despite the protective and restorative function of the mantle there can be no doubt that in some situations it is destructive of underlying bone, although this seems very mild in contrast with the fibrous tissue in many cases of osteoarthritis, rheumatoid arthritis and tuberculosis. There is very little information about the number, size, time of appearance and rate of development of the chondroid and fibrous foci at the surface of the exposed bone in osteoarthritis, and nothing about their effects on the results of osteotomy.

vol. 56 B, No. 2, MAY 1974

D 
In some of the cases the fibrous mantle extends over adjacent articular cartilage and in a proportion of these the evidence suggests that it replaces the cartilage, which again invites comparison with joint disease. The picture at this site is further complicated by an apparent conversion of surface cartilage cells to fibroblasts with the formation of a fibrous layer; this activity on the part of cartilage cells is not widely recognised but can be readily found in many situations if looked for.

Bone remodelling reduces the bone sclerosis and is accompanied by pronounced vascularity. Sokoloff (1969) refers to "innumerable publications formulating over-simplified generalisations about the effect of the circulation on atrophy and growth of bone". Despite a widely held view that osteolytic foci, so-called "cysts", are the result of the irruption of synovial fluid into the subchondral bone, implying that they are fluid-filled spaces, a large proportion are solid masses of fibrous tissue; others are fibrous tissue undergoing mucoid degeneration, and the remainder consist of fibrous tissue, mucoid degeneration and cystic space in a wide range of proportions. Communication of the lesion with the joint cavity is less constant than is suggested by Landells (1953). Their resolution is by membrane bone formation in the fibrous tissue, the cyst space if present being first filled in by fibrin and granulation tissue.

\section{SUMMARY}

1. Osteotomy for osteoarthritis of the hip induces a fibrin layer over the exposed bone which forms the basis of a fibrocellular protective mantle that can differentiate towards cartilage.

2. The process is accompanied by bone remodelling, which reduces sclerosis, resolves osteolytic foci and, in company with bone formation in the fibrous mantle, restores the subchondral plate. 3. Many important aspects of the pathogenesis of osteoarthritis and of its partial repair by osteotomy remain to be elucidated.

All but one of the cases reported on here were Mr K. I. Nissen's, and three specimens were obtained entirely through his interest. I would like to express my appreciation of the photographs and photomicrographs prepared by $\mathrm{Mr}$ T. R. Davis in the Department of Morbid Anatomy and of the histological sections prepared by the technicians.

\section{REFERENCES}

Bentley, G. (1974): Experimental osteoarthritis. In Normal and osteoarthritic articular cartilage. Institute of Orthopaedics, London. (In press.)

HarRIS, N. H., and KirWAN, E. (1964): The results of osteotomy for early primary osteoarthritis of the hip. Journal of Bone and Joint Surgery, 46-B, 477-487.

Landells, J. W. (1953): The bone cysts of osteoarthritis. Journal of Bone and Joint Surgery, 35-B, 643-649.

MEACHIM, G., and Osborne, G. V. (1970): Repair at the femoral articular surface in osteoarthritis of the hip. Journal of Pathology, 102, 1-8.

NisSEN, K. I. (1966): Osteotomy for the early arrest of idiopathic coxarthrosis. 10e Congrès de la Société Internationale de Chirurgie Orthopédique et de Traumatologie, p. 170.

NisSEN, K. I. (1971): The rationale of early osteotomy for idiopathic coxarthrosis (epichondro-osteoarthrosis of the hip). Clinical Orthopaedics, 77, 98-104.

Sokoloff, L. (1969): The Biology of Degenerative Joint Disease. Chicago and London: University of Chicago Press. 